\title{
FirstSteps: A mHealth tool for Children Registration and Monitoring
}

\author{
Jesuloluwa S. Eyitayo, Joke A. Badejo, Aderemi A. Atayero \\ Department of Electrical and Information Engineering Covenant University Canaanland-Ota, Nigeria \\ jileyitayo@gmail.com,joke.badejo@covenantuniversity.edu.ng, atayero@covenantuniversity.edu.ng
}

Abstract-The right of every child to survive, live healthy, have a voice and be educated begins with being uniquely identified immediately at birth. This paper details the development of a mHealth tool, coined FirstSteps, which incorporates an extensive registration platform for children under age five into a functional mobile child healthcare application. Considering diversity and cultural practices, FirstSteps adopts features (such as schedule setup for vaccination, doctor's appointment, recording child growth at various developmental stages) which can be deployed in sub-Saharan Africa and complements them with child registration records, one of the vital need of the region. It is expected that this will aid in achieving significant birth/child registration thereby providing quality data useful for reduction of high mortality rate in sub-Saharan Africa.

Keywords-Maternal and Child Health; mobile phones; Mobile Health (or mHealth); vaccination; smart and connected communities; child registration 